\title{
ARAPUCA light trap for large liquid argon time projection chambers
}

\author{
H. da Motta* \\ Author affiliation: Centro Brasileiro de Pesquisas Físicas (CBPF), R. Dr. Xavier Sigaud, 150 - \\ Urca, Rio de Janeiro - RJ, 22290-180, Brazil \\ E-mail: helio@cbpf.br
}

\section{A. A. Machado, L. Paulucci}

Affiliation: Universidade Federal do ABC (UFABC),

Av. dos Estados, 5001, Santo André, SP, 09210-170, Brazil

E-mail: ana.amelia@ufabc.edu.br ${ }^{\dagger}$

\section{E. Segreto, A. Fauth, B. Gelli, M. R. Guzzo, G. Marques}

Affiliation:Instituto de Física Gleb Wataghin, Universidade Estadual de Campinas - Unicamp, Rua Sergio Buarque de Holanda, No 777, CEP 13083-859 Campinas, SP, Brazil

E-mail: segreto@ifi.unicamp.br ${ }^{\ddagger}$

\section{Galante, V. Teixeira, W. Araujo, C. Ambrósio, M. Bissiano}

Affiliation: Laboratório NAcional de Luz Síncroton (LNLS),

Rua Giuseppe Máximo Scolfaro, 10000 - Guará, Campinas - SP, 13083-100, Brazil

\section{F. Marinho}

Affiliation: Universidade Federal de São Carlos,

Rodovia Anhanguera, km 174, 13604-900, Araras, SP, Brazil

\begin{abstract}
ARAPUCA is a totally innovative device for liquid argon scintillation light detection. It is composed of a passive light collector and of active devices. The active devices are standard SiPMs that operate at liquid argon temperature, while the passive collector is a photon trap that allows the collection of light with extremely high efficiency. The total detection efficiency of the device can be tuned by modifying the ratio between the area of the active components (SiPM) and that of the optical window. Few arrays of ARAPUCAs will be installed inside the prototype of the Deep Underground Neutrino Experiment - protoDUNE - and their performances will be compared with those of more standard solutions based on guiding bars. The results of the most recent tests of ARAPUCAs in a liquid argon environment, which led to the actual design for the protoDUNE, will be reported together with the proposal of a photon detection system for the Deep Underground Neutrino Experiment based on ARAPUCAs combined with dielectric mirror foils coated by wavelength-shifter.
\end{abstract}

The 19th International Workshop on Neutrinos from Accelerators-NUFACT2017

25-30 September, 2017

Uppsala University, Uppsala, Sweden

${ }^{*}$ Speaker.

${ }^{\dagger}$ Corresponding author.

‡Corresponding author. 


\section{Introduction}

Neutrino physics is actually recognized to be one of the most important tool to investigate new physics beyond the Standard Model. One of the most relevant experiments in this field is the Deep Underground Neutrino Experiment (DUNE), which is aimed to address some of the open questions in this field, namely the measurement of the $\mathrm{CP}$ violating phase in the leptonic sector, the hierarchy of neutrino masses and the $\theta_{23}$ octant.

The DUNE foresees the realization of a neutrino beam and of a near detector, both located at the Fermi National Accelerator Laboratory (Fermilab), Illinois, and a far detector based on the technology of Liquid Argon (LAr) TPC that will be installed at the Sanford Underground Research Facility in South Dakota, 1,300 km fa raway. This is the ideal distance since it will give the highest sensitivity in measuring the $\mathrm{CP}$ violating phase and in determining the mass hierarchy [1].

Moreover the huge active mass of the far detector will allow to develop a rich program of non accelerator physics, that includes the search for proton decay, the detection of supernova neutrinos and of atmospheric neutrinos.

The experimental technique foresees the detection of LAr scintillation light for various purposes. LAr is known to be an abundant scintillator, emitting 40 photons per $\mathrm{KeV}$ of deposited energy by minimum ionizing particles. Scintillation photons are emitted through the transition of the $A r_{2}^{*}$ excimer to the ground dissociative state made of two separate argon atoms. Unfortunately photons are emitted in the Vacuum Ultra Violet (VUV) with a wavelength of $128 \mathrm{~nm}$, where the vast majority of commercial photo-sensors is not sensitive. The most common and viable way to detect LAr VUV photons is to downconvert their wavelength to the visible through the use of wavelength shifting compounds which are coupled to standard (cryogenic) photo-sensitive devices with glass or fused silica window.

The scintillation light detection system for a massive LArTPC, like the one foreseen for the DUNE far detector, is very challenging since it needs to meet the discording requirements of having an extremely large coverage - given the internal dimensions of the detector - and a good detection efficiency, at the level of percent - to meet the physics goals of the experiment - while keeping the costs at an acceptable level.

One recent development for VUV photon detection in LAr is represented by the ARAPUCA device [2]. It is constituted by a light collector coupled to silicon photo-sensors (SiPM) used to read-out the collected photons. This configuration allows to increase the effective area of the SiPM array by a factor which can be higher than ten in optimal conditions.

The core of the ARAPUCA concept is represented by the light collector. It consists of a box cavity with internal highly reflective walls from which photons can reflect back and forth until being detected by the SiPM array (or absorbed). The acceptance window of the box is made by a short-pass dichroic filter ${ }^{1}$ coated with two different wavelength shifters, one on each side.

The external shifter converts the VUV photon coming form the LAr active volume to a wavelength below the cut-off, allowing it to cross the filter, while the internal one absorbs again the

\footnotetext{
${ }^{1}$ This particular type of filters has the properties of being highly transparent to photons with wavelength below a given cut-off, while being highly reflective to photons with wavelength above the same cut-off.
} 
converted photons and shifts its wavelength above the cut-off, where the filter is totally reflective. Since the box has reflective internal surfaces, the photon results to be trapped inside it.

For use in a liquid argon (LAr) environment the two wavelength shifting layers can be optimized as follows. Scintillation light is emitted by LAr with a wavelength of $127 \mathrm{~nm}$. The first shifter is para-terphenyl (pTP) [3], deposited on the external side of the acceptance window, which converts the liquid argon scintillation light to a photon of wavelength that peaks around $350 \mathrm{~nm}$, below the filter cutoff, chosen to be $\sim 400 \mathrm{~nm}$. After passing through the dichroic filter, the photon reaches the second shifter, TetraPhenyl-Butadiene (TPB) which shifts it to the visible region (peak $\sim 430 \mathrm{~nm}$ ) [4]. When entered the box, the photon will likely reflect a few times (including on the dichroic filter surface) before being detected by the SiPM array.

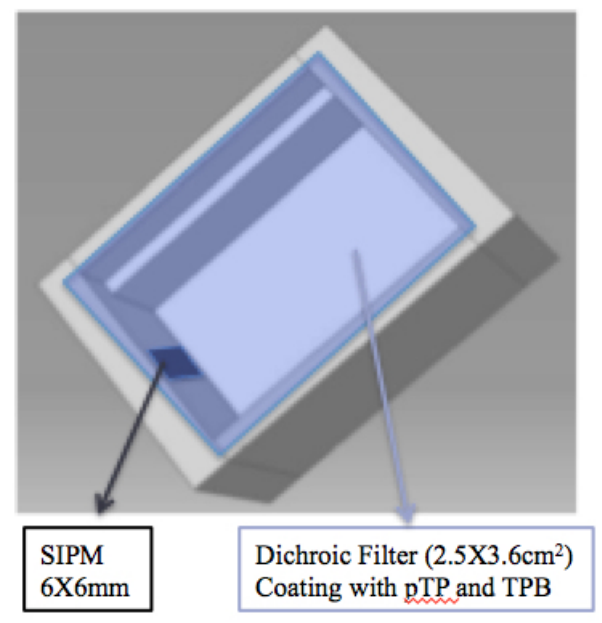

Figure 1: Schematic diagram of the ARAPUCA.

ARAPUCA provides a reasonably high light detection efficiency (few percent) on large areas (hundreds of $\mathrm{cm}^{2}$ ) with a limited coverage of active components (SiPM). These properties make it particularly suited to be used in large liquid argon Time Projection Chambers, like the ones of the DUNE experiment.

\section{Experimental Setup}

The tests of the ARAPUCA using liquid argon (LAr) were performed at the facilities of the Toroidal Grating Monochromator (TGM) beamline of the Brazilian Synchrotron Light Laboratory (LNLS). In order to observe the ARAPUCAś efficiency, the sensor was installed in a vacuum tight stainless steel cylinder filled with ultra pure LAr. Before filling, the chamber is evacuated down to a residual pressure of $10^{-6}$ mbar to avoid argon contamination. An alpha source was installed in front of the acceptance window of the ARAPUCA at a distance of $4 \mathrm{~cm}$, in correspondance of its geometrical center. The radioactive source is constituted by an ${ }^{238} \mathrm{U}-\mathrm{Al}$ alloy in the form of a metallic foil, which emits $\alpha$ particles with a continuous spectrum with an end-point of $4.267 \mathrm{MeV}$. The energy loss of the energetic particle in LAr was responsible for the production of the scintilla- 
tion ligth, which was collected and detected by the ARAPUCA. A schematic of the experimental setup is shown on figure 2 .

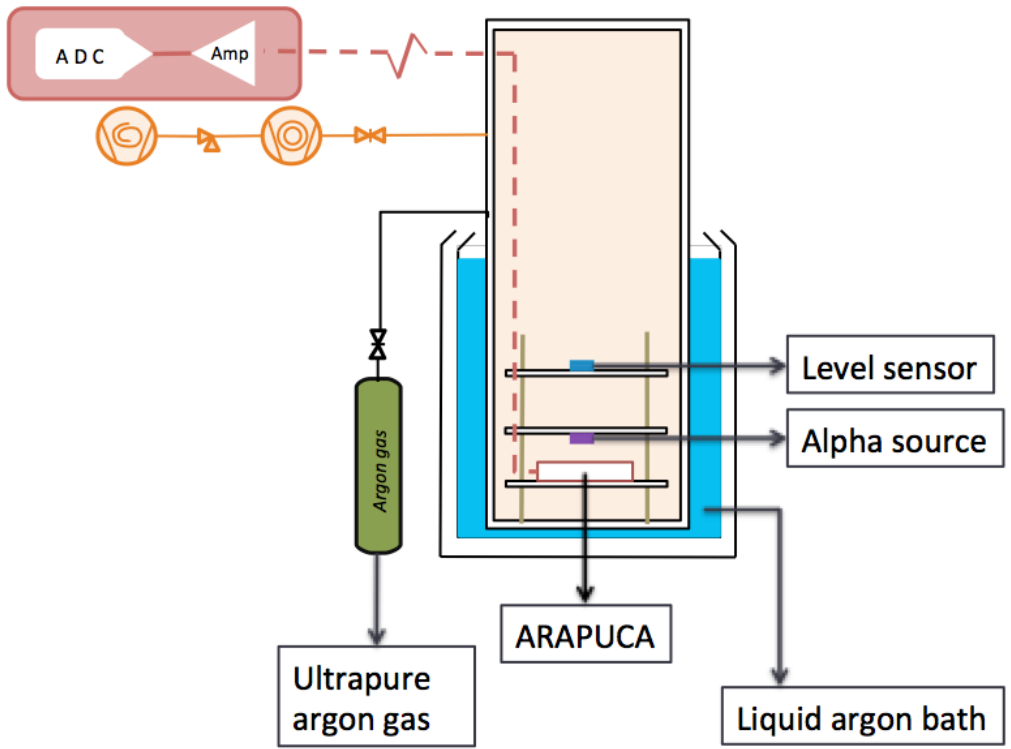

Figure 2: Schematic diagram of the vacuum chamber, complete with gas system and the ARAPUCA assembly, inside a dewar filled with liquid argon.

The alpha particle source has been characterized with the robust method of alpha particle spectrometry in a solid state detector. It consists in exposing the passive plastic detector to the radioactive source. The emitted alpha particles interact with the polymeric chains, causing damages and creating the so called etch pits. These pits have been demonstrated to be compatible with those produced by the $\alpha$ particles emitted by an ${ }^{238} \mathrm{U}$ source of infinite thickness. The signal coming from the SiPM is digitized (Acqiris, DP235 Dual-Channel PCI Digitizer Card - 1GS/s, 8 bit dynamic range) and stored on a computer.

\section{Data Analysis}

Recorded waveforms have been individually analyzed. In order to determine the Single Electron Response (SER) of the SiPM in LAr, single photon signals have been searched in the tail of the scintillation signals. An hit-find algorithm has been implemented, which searches for single, isolated peaks and integrates its charge over $300 \mathrm{~ns}$. The histogram of the integrals for a large number of isolated peaks is shown in figure 3 , right. The first peak is produced by one photon signals, while the others by multiple photons ( 3 more peaks are visible, due to two, three and four photons). The distribution has been fitted with a function made by four Gaussians. The mean value and standard deviation of the Gaussian distribution related to the single photon have been left as free parameters, while the mean value and standard deviation of the Gaussians distributions of multi-photons peaks 

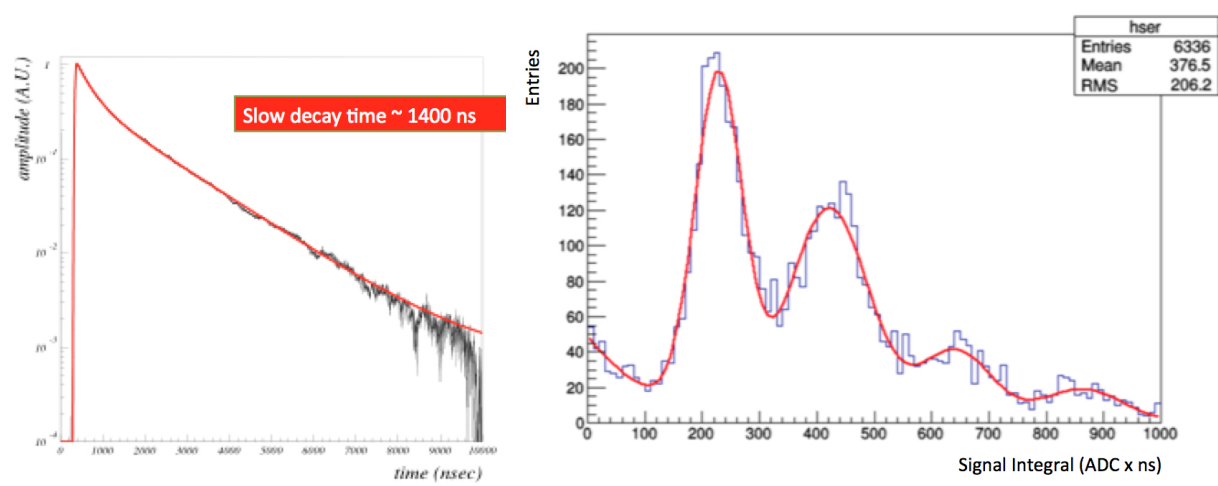

Figure 3: Left: Average waveform detected by the ARAPUCA, fast and slow components of the scintillation light are clearly visible. The fast component is degraded by the slow decay time of the SiPM. The slow component has a decay time of about $1400 \mathrm{~ns}$, as expected for ultra clean argon. Right: Single Electron Response of the SiPM used to read-out the photons trapped inside the ARAPUCA box. The first peak is due to genuine one photon singal, while the others are due toto multiple photons. Red line is the result of the fit of the histogram with a function which is constiuted by the sum of four non-independent gaussians (see text).

are not free and are given by the relations:

$$
\begin{gathered}
\bar{x}_{n}=n \times x_{0} \\
\sigma_{n}=\sqrt{n} \times \sigma_{0}
\end{gathered}
$$

where $\bar{x}_{n}$ is the mean value of the peak relative to the $n^{\text {th }}$ photon, $x_{0}$ is the mean value of the one photon peak, $\sigma_{n}$ is the standard deviation of the peak relative to the $n^{\text {th }}$ photon and $\sigma_{0}$ is the standard deviation of the one photon gaussian distribution. The normalization factor of each one of the peaks has been left as free parameter.

The result of the fit is shown in figure 3 , right with a red line. $x_{0}$ represents the value of the integral of a signal produced by one single photon and is the overall-calibration factor of the light sensor.

The total amount of photons detected by the SiPM in coincidence with an ionization event induced by the $\alpha$ particles emitted by the source is determined by integrating the signal starting form the $t_{0}$ (time corresponding to the trigger of the digitizing board) up to $t_{0}+10 \mu \mathrm{sec}$ and dividing it by the calibration factor $x_{0}$. The spectrum of the number of photons detected by the ARAPUCA is shown in figure 4. The spectrum has been fitted with a function which describes the expected energy spectrum of the residual energy of the $\alpha$ particles emerging from the source, determined through a dedicated Monte Carlo simulation. In fact, the number of photons detected by the ARAPUCA is proportional to the total number of photons produced by the $\alpha$ particle, through the fraction of the solid angle subtended by the ARAPUCA acceptance window, which is in turn proportional to the $\alpha$ 's residual energy. The end point of the spectrum in terms of photo-electrons comes from the fitting procedure and lies approximately in the middle of the high energy descending part of the 


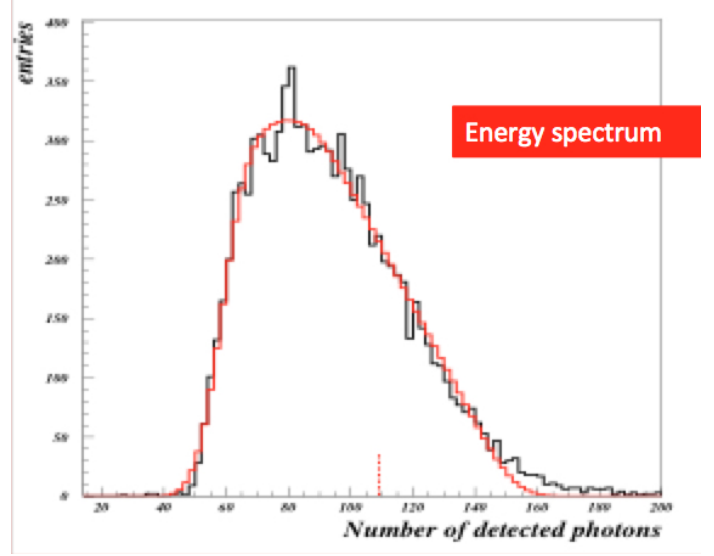

Figure 4: Spectrum of the integrated scintillation signals (black line). The experimental spectrum has been fitted with the source energy spectrum predicted by a dedicated Monte Carlo simulation (red line).

spectrum, indicated by a red line in figure 4. Its value results to be of $109 \pm 1$ photo-electrons. The number of photons impinging on the acceptance window of the ARAPUCA ca be calculated through simple geometrical considerations. The total amount of photons emitted by $\alpha$ paricles with energies corresponding to the end point of the spectrum is given by:

$$
N_{p h}=N_{\text {Lar }}^{0} \times 4.3 \mathrm{MeV} \times Q_{\alpha}
$$

where $N_{0}$ is the ideal photon yield in $\mathrm{LAr}$ and is equal to $\sim 5.1 \times 10^{4}[6]$ photons/MeV and $Q_{\alpha}$ is the quenching factor for $\alpha$ particles, which is known to be 0.7 [7]. Photons are emitted isotropically and the fraction which hit the ARAPUCA window is given by the solid angle it subtends. It can be analytically calculated because of the very simple geometry of the system with the equation:

$$
\Omega=4 \arctan \frac{a b}{2 d \sqrt{4 d^{2}+a^{2}+b^{2}}}
$$

where $\mathrm{a}$ and $\mathrm{b}$ are the linear dimensions of the acceptance window $\left(2.5 \times 3.6 \mathrm{~cm}^{2}\right)$ and $\mathrm{d}$ is the distance of the source $(4 \mathrm{~cm})$. The solid angle subtended by the ARAPUCA window is approximately 0.49 rad and the fraction, $\mathrm{f}$, of photons impinging on the ARAPUCA window is $3.9 \%$. The detection efficiency of the ARAPUCA is estimated by dividing the number of photoelectrons detected in correspondence of the end point of the $\alpha$ spectrum by the number of photon impinging on its window, $\mathrm{N}_{p h} \times \mathrm{f}$, and results to be:

$$
\varepsilon=1.8 \% \pm 0.3 \%
$$

The average waveform of the signals is shown in figure 3, left. Fast and slow components of scintillation light are clearly visible. The fast component decay time is dominated by the SiPM response, while the slow one by the scintillation light whose value of $1400 \mathrm{~ns}$ is compatible with pure liquid argon [8] [9]. 


\section{Monte Carlo Simulations}

The Geant4 toolkit [5] was used to simulate the whole device. The reflection properties of the teflon used in the box were implemented along with the ones for the three layers of the acceptance window. A view of the whole simulated device is seen in figure 5. The dimensions and geometry were chosen to reproduce the prototype. It has internal dimensions of $36 \mathrm{~mm}$ x $25 \mathrm{~mm}$ x $6 \mathrm{~mm}$ (length $\times$ width $\times$ height) and a teflon thickness of $1 \mathrm{~cm}$ with $95 \%$ reflectivity for visible light. The SiPM has an active surface of $6 \times 6 \mathrm{~mm}^{2}$ and a quantum efficiency of $25 \%$. Roughly half of the incident photons are emitted back when converted by the PTP while the remaining photons are effectively trapped inside the ARAPUCA. Some of them reach the SiPM after a few reflections (2-3) while the remaining are either detected after many reflections or absorbed by the surface. The simulation predicted a detection efficiency of $1.7 \pm 0.1 \%$, well compatible with the experimental result.

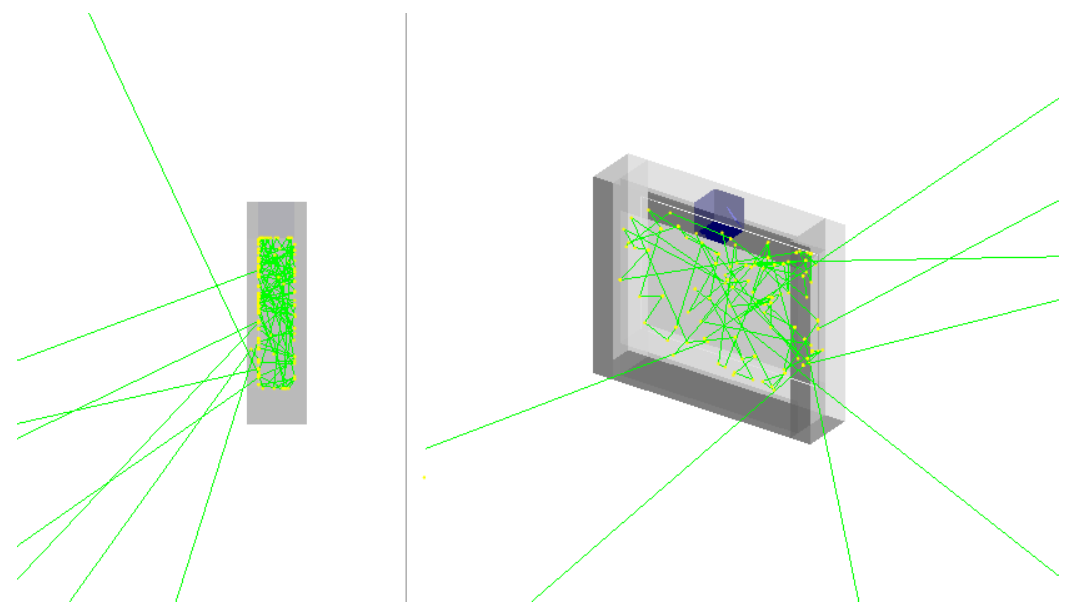

Figure 5: Two views of the simulated ARAPUCA device: lateral (left) and rotated (right). The SiPM (blue) is located on the top. The box is made of highly reflective teflon (gray). Photons are shown as green lines.

\section{Conclusions}

The measured efficiency of the ARAPUCA with $9 \mathrm{~cm}^{2}$ active area was $1.8 \% \pm 0.3 \%$. This value is in good agreement with the Monte Carlo simulation, which gives a value of $1.7 \% \pm 0.1 \%$. The ARAPUCA device promises to have a high detection efficiency for low energy events (supernova neutrino and proton decay) in huge LArTPC detectors (two of the three main goals of DUNE experiment). R\&D for ARAPUCA is ongoing, not only in Brazil but also in USA, Europe and other Latin America Countries.

\section{References}

[1] DUNE/LBNE/LBNF Collaboration, Baseline optimization for the measurement of CP violation and mass hierarchy in a long-baseline neutrino oscillation experiment, arXiv:1311.0212 [hep-ex], 2013. 
[2] A.A. Machado and E. Segreto, Journal of Instrumentation, 11, C02004 (2016).

[3] T.A. DeVol et al., Evaluation of p-terphenyl and 2,2" dimethyl-p-terphenyl as wavelength shifters for barium fluoride, Nucl. Instrum. Meth. A 327 (1993) 354.

[4] R. Francini et al., VUV-Vis optical characterization of Tetraphenyl-butadiene films on glass and specular reflector substrates from room to liquid Argon temperature, JINST 8 P09006 (2013).

[5] S. Agostinelli et al., Nuclear Instruments and Methods in Physics Research Section A, 506, 250-303 (2003).

[6] T. Doke et al., Nuclear Instrument and Methods in Physics Research Section A, 291 617-620 (1990).

[7] T. Doke et al., Nuclear Instrument and Methods in Physics Research Section A, 269291 (1988).

[8] R. Acciarri et al. 2010 JINST 5 P06003.

[9] R. Acciarri et al. 2010 JINST 5 P05003. 